International Journal of Food Science, Nutrition and Dietetics (IJFS)

ISSN 2326-3350

\title{
Preparation and Characteristic of Dextran-BSA Antibody and Establishment of it's Elisa Immunoassay
}

Zhen-ming $\mathrm{X}^{1 *}$, Li-sha $\mathrm{F}^{1}$, Da-feng $\mathrm{L}^{1,2}$, Lin $\mathrm{Y}^{1}$

Research Article

${ }^{1}$ Faculty of Light and Chemical Engineering, Guangdong University of Technology, Guangzhou 510060, China.

${ }^{2}$ Guangzhou Institute of Sucrose, Guangzhou 510316, China.

\begin{abstract}
The enzyme linked immunosorbent assay (ELISA) is a potential tool for the determination of dextran. In this study, dextran-BSA antigens were prepared by Reductive amination method, and were confirmed by SDS-PAGE and free amino detection. The effects of coupled reaction conditions such as different oxidation degree of dextran, the reaction time were investigated and the immunity of the resulting dextranBSA neoglycoprotein antigens were evaluated through the interaction with standard dextran antibody. The immunogen was immunized with white rabbits to obtained polyclonal antibody respectively. A general and broad class-specific Elisa detection method was developed according to Elisa theory. The method was put to use for quantitative analysis of dextran in practical saccharose samples.
\end{abstract}

Keywords: Dextran; Neoglycoprotein Antigen; Preparation; Polyclonal Antibody; ELISA.

\section{*Corresponding Author:}

Xie Zhen-ming

Faculty of Light and Chemical Engineering, Guangdong University of Technology, Guangzhou 510060, China.

E-mail: raretea@126.com

Received: May 06, 2014

Accepted: June 10, 2014

Published: June 12, 2014

Citation: Zhen-ming X, Li-sha F, Da-feng L, Lin Y (2014) Preparation and Characteristic of Dextran-BSA Antibody and Establishment of it's Elisa Immunoassay. Int J Food Sci Nutr Diet. 3(6), 122-128. doi: http:/ / dx.doi.org/10.19070/2326-3350-1400024

Copyright: Zhen-ming $\mathbf{X}^{\circ} 2014$ This is an open-access article distributed under the terms of the Creative Commons Attribution License, which permits unrestricted use, distribution and reproduction in any medium, provided the original author and source are credited.

\section{Introduction}

Dextrans are high-molecular-weight straight chain polysaccharides synthesized from sucrose by dextran sucrases. In sugar production, formation of dextran not only causes severe economic losses due to sugar loss, but also causes processing problems including high viscosity, poor filtration, low evaporation rate, poor crystallization, and slow mud settling [1-3]. The use of the glycosyl hydrolase is the most efficient method to hydrolyze dextrans at sugar factory. However, due to the high price of dextranase, dextran needs to be exactly quantitated in order to decide the precise amount of dextranase required for the hydrolyzation treatment in syrup [4-5].

Several different methods are commonly used for the determination of dextran in sugar industry including Haze method [6], Roberts copper method [7], ASI II method [8] and the improved alcohol Haze method. However, these methods, except ASI II, are not specific for the determination of dextran and may cross react with other polysaccharides coexisting. Two new methods,
Midland SucroTest TM and Optical Activity Ltd. DASA method have been reported to detect dextran only. But the DASA method might give erroneously high levels of dextran, due to either partial dextran hydrolysis or poor filtration [9]. Midland SucroTest TM is an immunological method that uses a monoclonal antibody to produce a linear increase in turbidity in response to dextran. This method is a rapid test that utilizes a minimum of equipments, and is highly specific to dextran, but the cost per analysis is considered to be too high for extensive use [10].

The enzyme linked immunosorbent assay (ELISA) has been developed for the detection and quantification of trace impurities in biotechnological production, which is rapid, efficient and selective [11-12]. Thus it will be of great significance to establish a low-cost ELISA analysis for dextran. The development of ELISA assay demands a sensitive antigen which could recognize the specific antibody produced by the animal's immune system. Dextran is a high molecular compound with low immunocompetent, thus it is necessary to add an appended immunocompetent part such as protein or biotin to dextran [13-15].

The aim of this study was to synthesize and analyze dextran-BSA conjugates and illustrate their characteristics as immobilized antigens on the surfaces of ELISA plates. The effects of synthesis conditions were investigated, resulting in different dextran-BSA antigens. Immunity and application of the optimal dextran-BSA antigen were also examined. Polyclonal antibody was obtained by means of animal immunization and the Elisa determination method was established for dextran detection. The specificity and accuracy of the method were examined and the Elisa detection method was used for dextran analysis in practical saccharose samples.

\section{Materials and Methods}

\section{Materials and reagents}

Dextran $(10 \mathrm{kDa}, 40 \mathrm{kDa}, 100 \mathrm{kDa}$ and $2000 \mathrm{kDa}$ as T10, T40, 
T100 and T2000) and bovine albumin serum (BSA) were from Sangon Ltd. (China), anti-dextran mouse monoclonal antibody clone DX-1 was obtained from StemCell Technologies, and other standard protein was purchased from Takara Biotechnology (Dalian) Company. Sheep anti-mouse-IgG-HRP, phosphate buffered saline (PBS), Tween 20 and TMB were supplied by Sigma (St. Louis, MO, USA) for immunoassay. Sodium periodate Merck, Germany), O-phthaldialdehyde (OPA), Coomassie brilliant blue R-250, basic fuchsin were supplied by (Sigma, USA), and all the other chemicals were of analytical grade (purity $>98 \%$ ). Deionized water (electrical conductivity $<2 \mu \mathrm{S} \mathrm{cm}^{-1}$ ) was used throughout the experiments. Ultra-15 hydrophilic ultrafilter tubes were supplied by Millipore (USA).

\section{Synthesis of dextran-BSA antigen}

Preparation of aldehyde-dextran: Aldehyde-dextran was obtained by oxidating dextran with sodium periodate, and different oxidization ratio (Dex/ $\mathrm{NaIO}_{4}$ weight ratio of 1/10, 1/60, 1/120 and $1 / 187$ ) were evaluated to optimize the preparation reaction. The reaction was stopped by adding ethylene glycol and the solution was ultra-filtered to remove the impurities and then freeze dried.

Preparation of dextran-BSA antigen: BSA was coupled with aldehyde-dextran according to the scheme presented in Figure 1. Different aspects were evaluated to optimize the reaction, including the reaction time $(12,24,48$ or $72 \mathrm{~h}$ ) and the different dextran molecular (T10, T10, T100, T2000). Sodium cyanoborohydride solution was added to terminate the reaction and the solutions were centrifuged at $5000 \mathrm{rpm}$ for $30 \mathrm{~min}$ using ultrafilter tubes to remove the impurity and the filtrate was stored at $4^{\circ} \mathrm{C}$.

\section{Characteristic of dextran-BSA antigen}

SDS-PAGE characterization : The resultant dextran-BSA conjugates were analyzed using the SDS-Polyacrylamide gel electrophoresis (SDS-PAGE) technique to confirm the presence of neoglycoprotein antigens. SDS-PAGE was performed according to the method of Laemmli[16] using 7.5\% acrylamide as separating gel and 3\% stacking gel containing $0.1 \%$ SDS. The protein and neoglycoproteins were prepared in a $0.08 \mathrm{M}$ Tris- $\mathrm{HCl}$ buffer at pH 6.8 containing 4\% SDS and 0.5\% mercaptoethanol. Electrophoresis was carried out at a constant voltage of $80 \mathrm{~V}$ for $4 \mathrm{~h}$. The gel sheets were stained with $0.2 \%$ Coomassie brillant blue R-250 for protein or with $0.4 \%$ periodate-fuchsin solution containing $0.2 \%$ basic fuchsin for neoglycoproteins conjugates [17]. A Nikon digital camera was used to obtain photographs.

Determination of free amino groups: The amount of free amino groups was measured using OPA method as described by Nielsen[18]. All spectrophotometer readings were performed at $340 \mathrm{~nm}$. Lysine was used as the standard for calibration curve and deionized water was used as reference. The free amino groups of modified dextran antigen and unmodified BSA protein were measured as $\mathrm{Ct}$ and C0. The degree of graft (DG) was calculated as follow:

$\mathrm{DG}=\left(\mathrm{C}_{0}-\mathrm{C}_{\mathrm{t}}\right) / \mathrm{C}_{0} \times 100 \%$

The immunocompetence analysis of antigen: The immunocompetence of dextran-BSA neoglycoprotein antigens were determined by indirect ELISA. The gained neoglycoprotein antigens were diluted in coating buffer $(0.05 \mathrm{M}$ carbonate, $\mathrm{pH} 9.6)$ to the specific concentrations for adsorption to the polystyrene microtiter plates $(100 \mu \mathrm{L}$ per well; an overnight incubation at $\left.4^{\circ} \mathrm{C}\right)$. At the end of the incubation, the plates were washed three times with phosphate buffered saline (PBS, $0.01 \mathrm{M}$ ) containing $1 \%$ (v/v) Tween 20. The primary and secondary antibodies (antidextran mouse monoclonal antibody clone DX-1 and Fc-specific sheep anti-mouse-IgG-HRP) were diluted in blocking solution and incubated on the plate at $37^{\circ} \mathrm{C}$ for $45 \mathrm{~min}$. After washing the plate, the reaction was developed by adding TMB to the wells and stopped by adding $0.5 \mathrm{M} \mathrm{H}_{2} \mathrm{SO}_{4}$. The absorption of the solution was measured at $450 \mathrm{~nm}$ on 1420 VICTOR D Multilabel Counter.

\section{Antibody synthesis and purification}

Male New Zealand White rabbits were immunized by subcutaneous injection to gain dextran-BSA antibody. Briefly, $500 \mu \mathrm{g}$ of the dextran T40-BSA antigen mixed 1:1 with Freund's complete adjuvant was injected into rabbits, and booster injections were administered twice at 2-week intervals with the same amount of antigen mixed 1:1 with incomplete Freund's adjuvant. Serum was collected and purified according saturation ammonium sulfate method and stored at $-20^{\circ} \mathrm{C}$.

\section{Establishment of dextran Elisa detection and practical sam- ple determination}

The dextran-BSA polyclonal antibody was analyzed and charactered by indirect non-competitive Elisa. The aspects as coating antigen concentration, the incubation time of antibody to enzyme-labeled antibody, the blocking solvent and the optimal antibody concentration were studied.

With the optimized Elisa condition, an indirect competitive Elisa procedure was performed as the method of Tijssen [19] for the determination of dextran. The linear calibration graph was plotted by the absorption of the reacted solution at $450 \mathrm{~nm}$ versus the concentration of dextran-BSA antibody. The slope and correla-

Figure 1. Schematic representation of the synthesis of dextran-BSA neoglycoprotein.

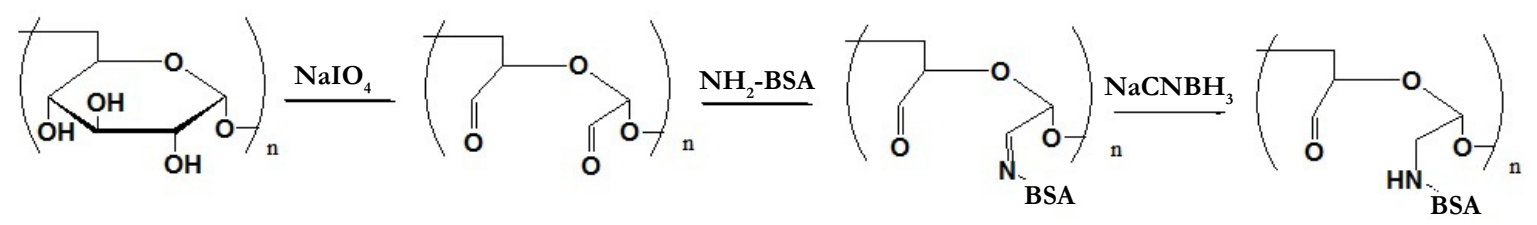


tion coefficient $(\mathrm{r})$ were calculated using Origin software, and the median inhibitory concentration $\mathrm{IC}_{50}$ and the detection limit were defined respectively. The specificity of the established Elisa detection method of dextran T40 to other different molecular dextran was evaluated by Cross reactivity (CR) as follow:

$\mathrm{CR}(\%)=\mathrm{IC}_{50}$ of dextran $\mathrm{T} 40 / \mathrm{IC}_{50}$ of other molacular dex$\operatorname{tran} \times 100 \%$

The practical applicability of the established Elisa detection method was demonstrated by quantitative determination of dextran T40 in saccharose sample.

\section{Results and Discussion}

\section{Synthesis and characteristic of dextran-BSA antigen}

Dextran-BSA antigen was synthesis by periodate oxidation of dextran (T10, T40, T100, T2000), then conjugated with amino groups of BSA and finally reduced by sodium cyanoborohydride. Different aspects on reaction condition were studied as follow.
The effect of oxidation rate to antigen: The effect of $\mathrm{NaIO}_{4}$ on the extent of oxidation was subsequently assessed. Dextran (e.g. T40) was oxidized with different weight ratios of Dex/ $\mathrm{NaIO}_{4}(1 / 10,1 / 60,1 / 120$ and 1/187). The SDS-PAGE result (figure 2) and DG analysis (figure 3) were shown. It was noted that, with increasing amount of $\mathrm{NaIO}_{4}$, dextran-BSA neoglycoprotein antigen was increased, and the grafting degree was also increased. In consideration of the strong oxidant can destroy the dextran chain, which likely reduced the immunocompetence of dextran-BSA antigen, and the strong oxidant expend the sodium cyanoborohydride during the Schiff's base reducing reaction. The Dex $/ \mathrm{NaIO}_{4}$ oxidation rate of $1 / 120$ was selected.

An ELISA assay was developed to study the interaction of the dextran-BSA antigen with anti-dextran mouse monoclonal antibody clone DX-1, and the immunocompetence of dextran-BSA antigens were evaluated and selected.

The dextran-BSA antigens obtained with different weight oxidation ratio (Dex $/ \mathrm{NaIO}_{4}=1 / 10,1 / 60,1 / 120$ and $\left.1 / 187\right)$ were combined with standard clone DX-1 antibody at a concentration of $2.5 \mu \mathrm{g} \mathrm{mL}^{-1}$, and the absorbance at $450 \mathrm{~nm}$ was monitored ( Figure 4). The results showed that the interaction of Dex-BSA

Figure 3. The effect of different degrees of oxidation on

a Coomassie Brilliant Blue R250; b periodic acid-fuchsine $1 \mathrm{BSA} ; 2 \mathrm{~T} 40 / \mathrm{NaIO}_{4}=1 / 10 ; 3 \mathrm{~T} 40 / / \mathrm{NaIO}_{4}=1 / 60 ; 4 \mathrm{~T} 40 / / \mathrm{NaIO}_{4}$ $=1 / 120 ; 5 \mathrm{~T} 40 / / \mathrm{NaIO}_{4}=1 / 187^{\circ}$

Figure 4. The OD value of different oxidized antigens combined with standard antibody

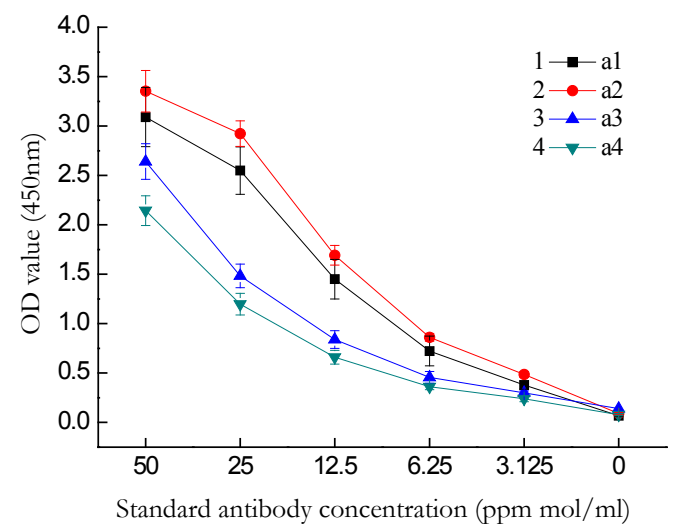

$\left(\mathrm{a} 1, \mathrm{a} 2, \mathrm{a} 3, \mathrm{a} 4\right.$ represent $\left.\mathrm{n}_{\mathrm{T} 40} / \mathrm{n}_{\mathrm{NaIO}}=1 / 10,1 / 60,1 / 120,1 / 187\right)$
DG of dextran-BSA antigen.

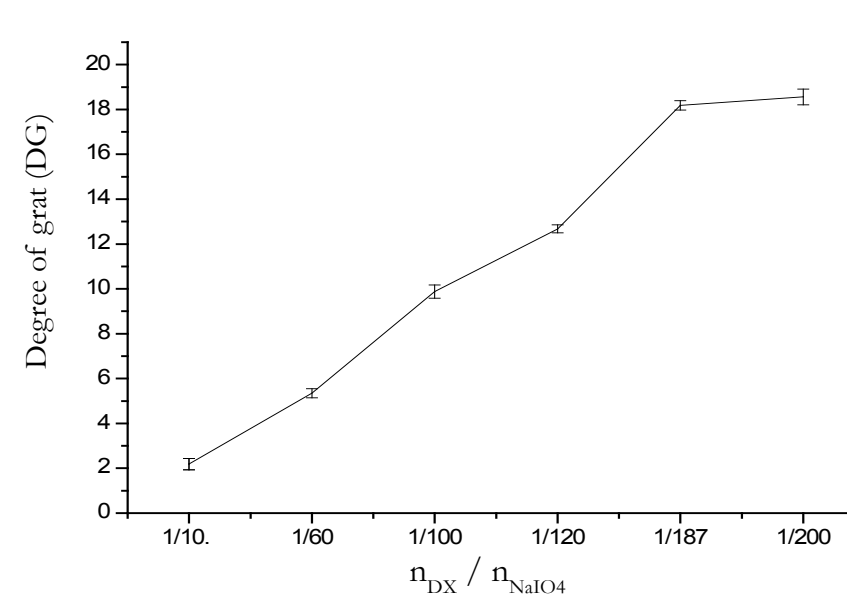

Figure 5. SDS-PAGE of dextran-BSA neoglycoprotein prepared in different Reaction time

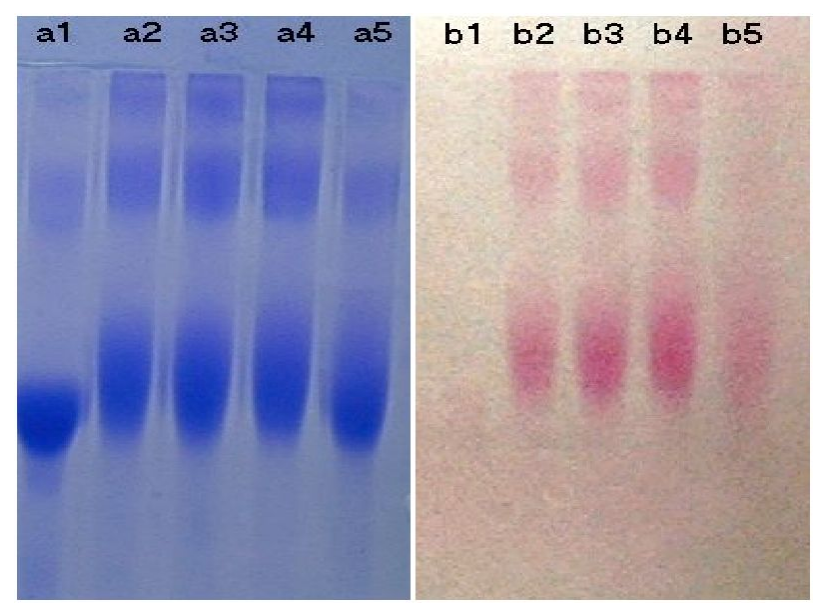

a Coomassie Brilliant Blue R250; b periodic acid-fuchsine 1 BSA $212 \mathrm{~h} ; 324 \mathrm{~h} ; 4$ 48h; 5 72h 
Figure 6. The effect of Reaction time on DG of dextranBSA neoglycoprotein

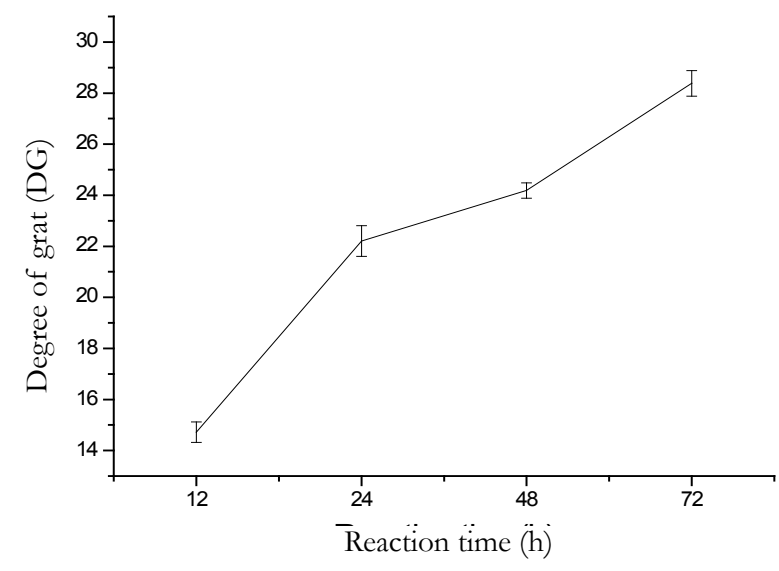

Figure 8. SDS-PAGE of dex-BSA antigens prepared from different sizes of dextrans.

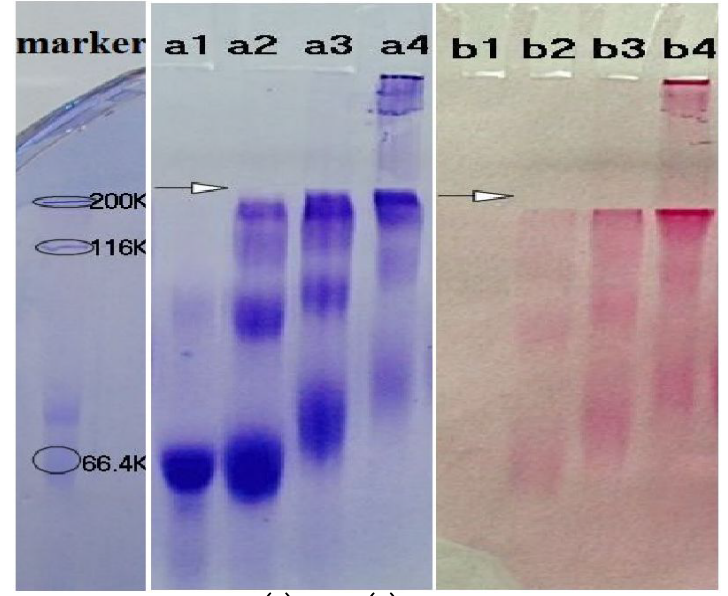

(a) (b)

a Coomassie Brilliant Blue R250; b periodic acid-fuchsine 1 T10; 2 T40; 3 T100; 4 T2000

Figure 10. Determination for optimal concentrations of coated antigen

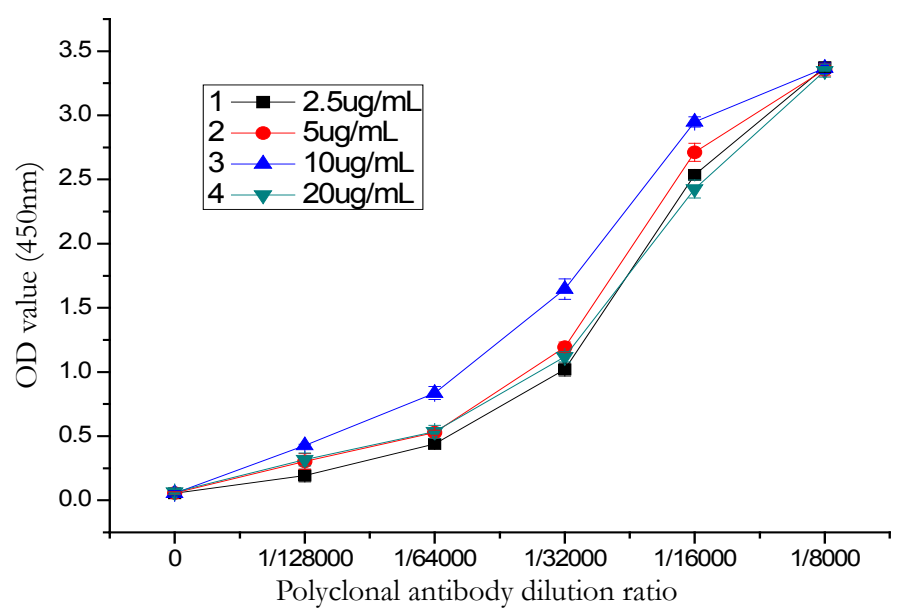

Figure 7. The OD value of different coupled reaction time of antigen combined with standard antibody

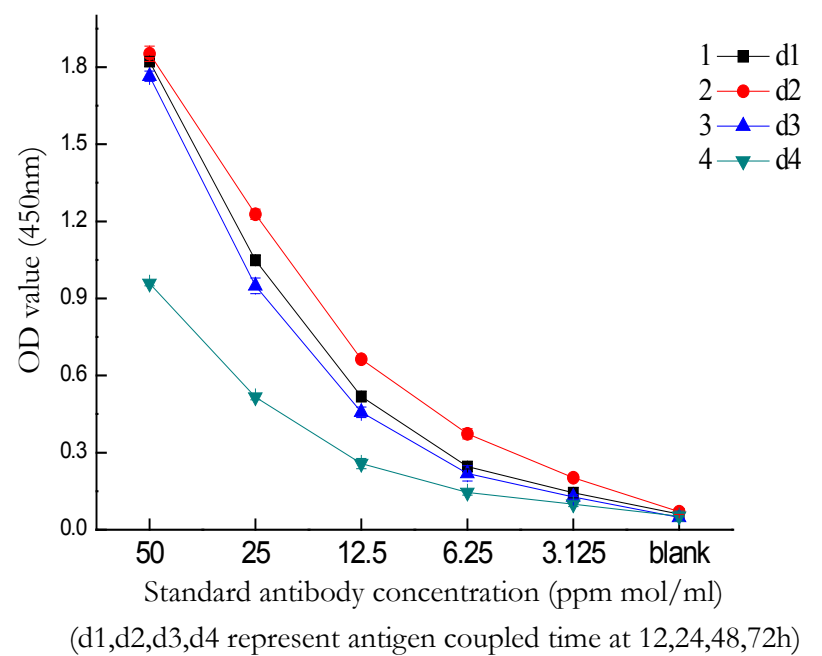

Figure 9. The OD value of different dextran antigen combined with standard dextran antibody

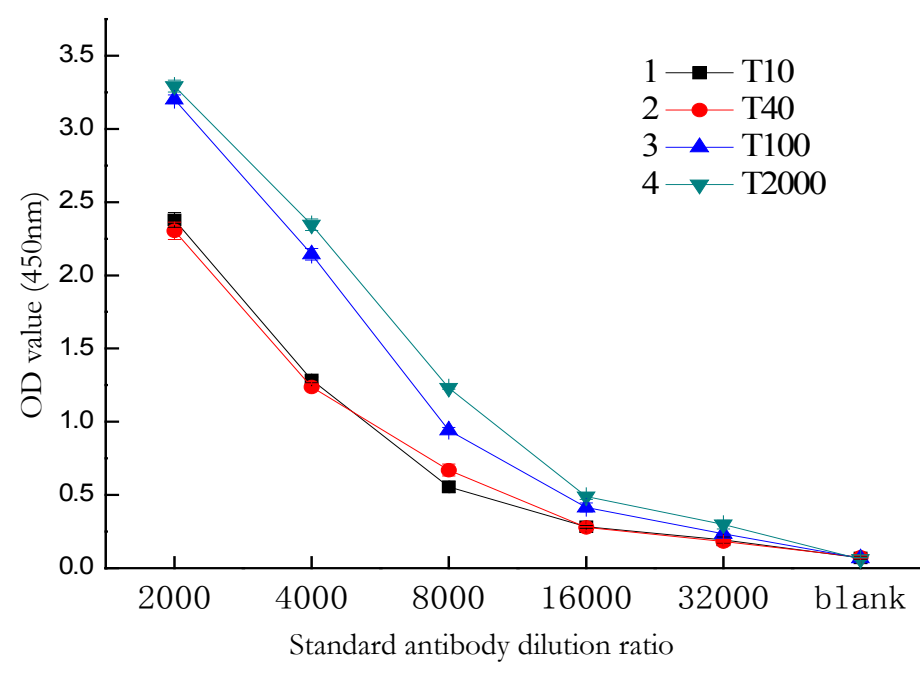

Figure11. Determination for optimal time of incubation

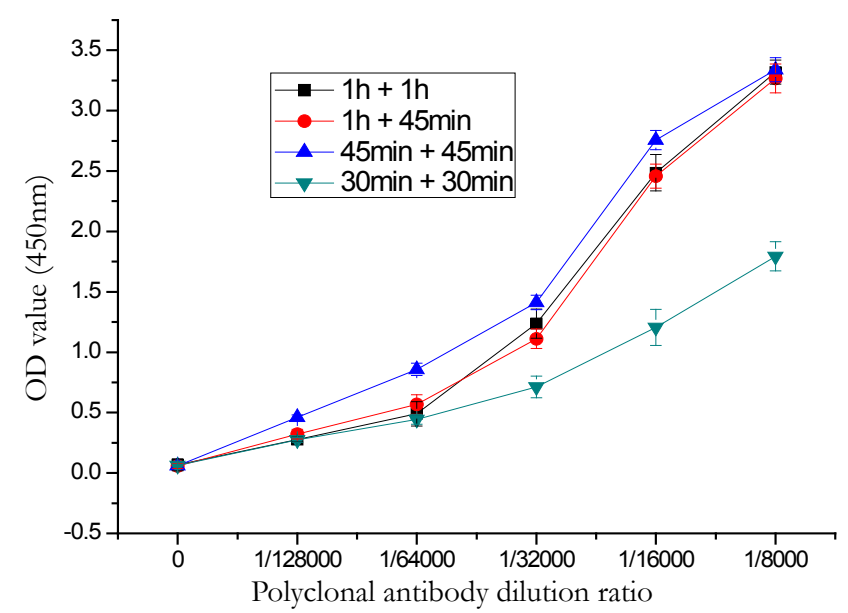


antigen (obtained with $\mathrm{Dex} / \mathrm{NaIO}_{4}$ weight ratio of $1 / 60$ ) with Clone DX-1 antibody was the strongest. This might be attributed to the fact that dextran with low degree of oxidation might reduce the coupling of BSA with dextran, which inhibited the interaction with Clone DX-1; while dextran with high degree of oxidation might destroy the dextran chains, and the increased BSA conjugated with dextran caused steric hindrance, which may also hinder the interaction.

The effect of reaction time to antigen: The time required for the completion of the coupled reaction was also assessed. The resulting dextran-BSA neoglycoprotein antigen (e.g. T40) obtained with different reaction times (12h, 24h, 48h and $72 \mathrm{~h}$ ) were analyzed on SDS-PAGE (Figure 5). It was found that the yield reached maximum at $24 \mathrm{~h}$ and kept a plateau, and the neoglycoprotein reduced at $72 \mathrm{~h}$. The degree of grafting (DG) was increased gradually, which was not consistent with the results observed on SDS-PAGE (Figure 6). The antigen reduced at $72 \mathrm{~h}$ might be the denaturation of neoglycoprotein with longer reaction time. The increased DG indicated only the decrease of free amino groups, and the performance of dextran-BSA antigens could not be evaluated simply by DG. Overall consideration, the time for the conjugation reaction was set at $24 \mathrm{~h}$.
At the coated antigen concentration of $10 \mu \mathrm{g} \mathrm{mL}^{-1}$, the interaction of dextran-BSA neoglycoprotein antigens (obtained after 12, 24, 48 and $72 \mathrm{~h}$ reaction) and Clone DX-1 were studied (figure 7). The OD450 absorbance shown the conjugate obtained after $24 \mathrm{~h}$ exhibited strongest interaction with Clone DX-1.

The effect of dextran molecular to antigen: Further studies on the coupling of BSA with different sizes of dextran (Figure 8) indicated that the resulting conjugates were different. As expected, higher molecular dextran obtained higher molecular dex-BSA antigen, which shown the conjugation reaction was dependent on the degree of oxidation, not on the molecular weight of dextrans.

A coated antigen concentration of $10 \mu \mathrm{g} \mathrm{mL}^{-1}$ was selected for the investigation of the interaction between Clone DX-1 antibody with dex-BSA antigen synthezed by different molecular of dextran. As shown in Figure 9, antigen with higher molecular dextran had strong interaction with Clone DX-1 antibody. This might be due to the enhanced selectivity and particularity of dextran-BSA antigen caused by high molecular dextran. As we preconceived, dextran antigen with high molecular and complex structure lead to higher immunological competence.
Figure12. Determination for optimal blocking reagents

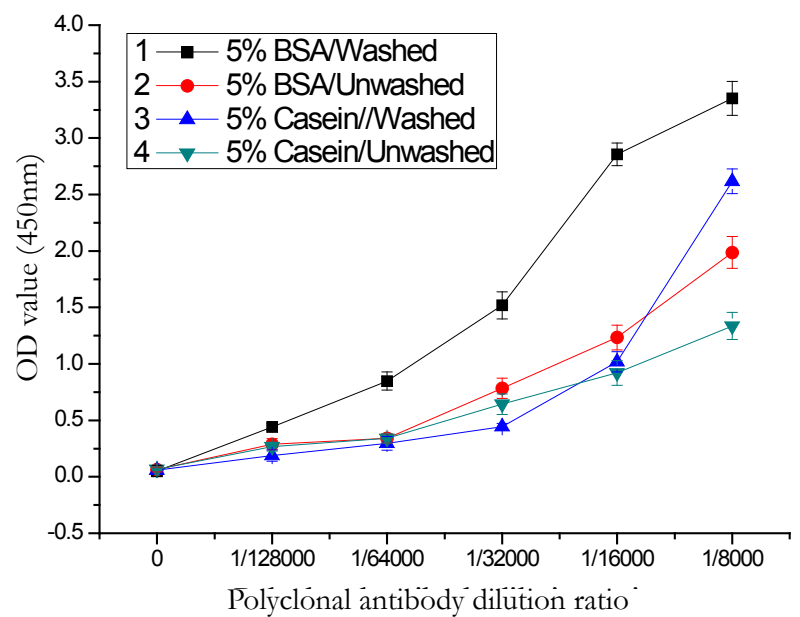

Figure14. The Inhibitory effect of different molecular dextran

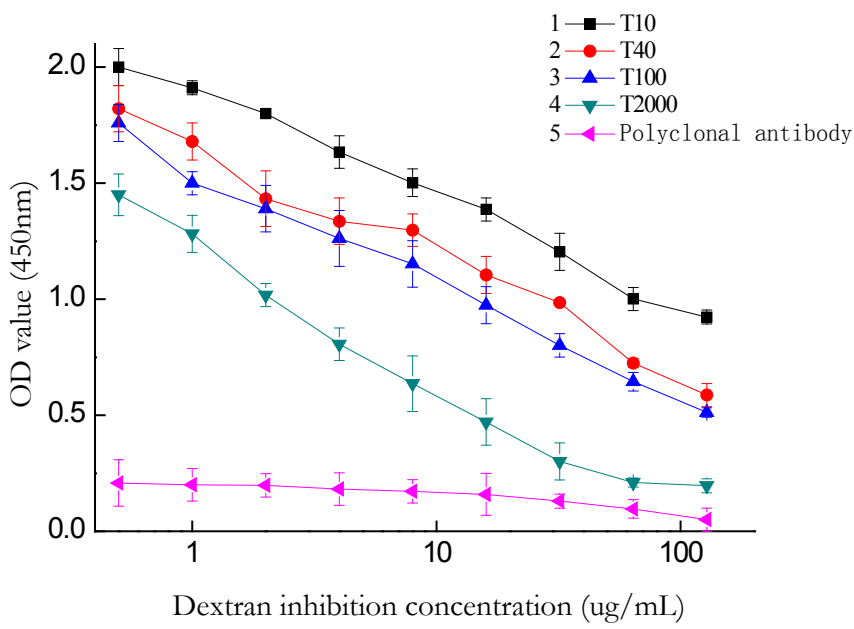

Dextran inhibition concentration $(\mathrm{ug} / \mathrm{mL})$
Figure13. Determination of the optimal dilution of polyclonal antibody

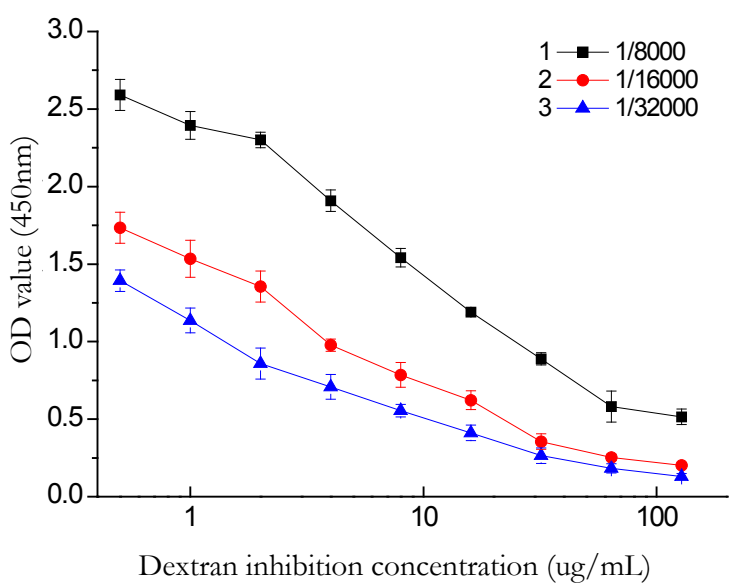

Figure15. The Inhibitory effect of antibody to Dextran T40 in practical sucros sample

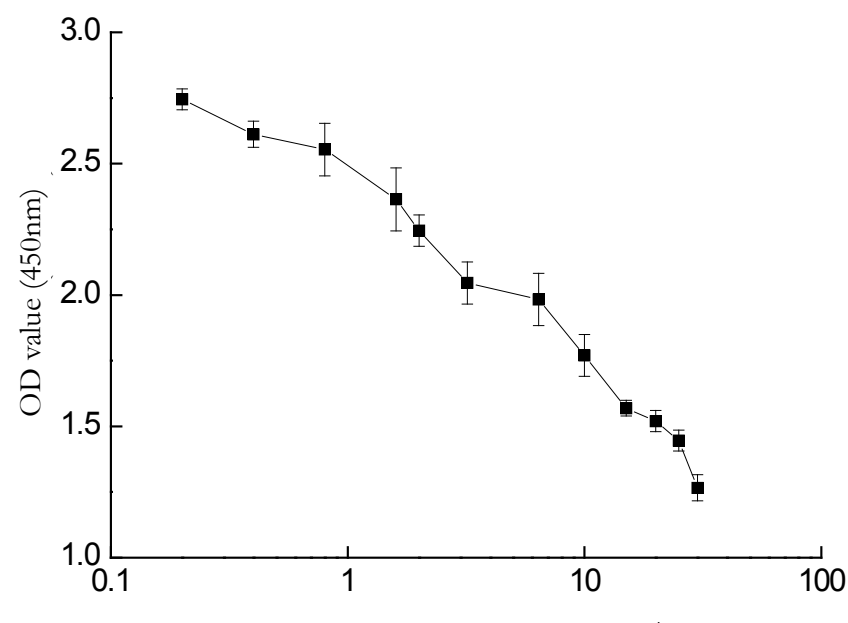

Dextran inhibition concentration $(\mathrm{ug} / \mathrm{mL})$ 
Table 1. Indirect competitive Elisa standard curve for different dextran

\begin{tabular}{|l|l|l|}
\hline Dextran & IC $_{50} / \mathbf{~ m g} / \mathrm{L}$ & CR \% \\
\hline T10 & 48 & 150 \\
\hline T100 & 8 & 25 \\
\hline T2000 & 4 & 12.5 \\
\hline Polyclonal antibody & 0 & 0 \\
\hline
\end{tabular}

\section{Preparation and immunity analysis of antibody}

The dextran T40 polyclonal antibody was prepared and purified as described in 2.4, and the indirect non-competitive Elisa for antibody characteristic was developed. The aspects as coating antigen concentration, the incubation time of antibody to enzymelabeled antibody, the blocking solvent and the optimun antibody concentration were studied as followed.

Optimization of the coating concentration: The dextranBSA antigen (e.g. T40, Dex $/ \mathrm{NaIO}_{4}$ ratio of $1 / 120$ ) was prepared as coating buffer at different concentrations of 2.5, 5, 10 and 20 $\mu \mathrm{g} \mathrm{mL} \mathrm{m}^{-1}$, respectively, and combined with dextran T40 polyclonal antibody for multiple proportion dilution Elisa analysis. According to immunoassay analysis mentioned above, the absorbance at $450 \mathrm{~nm}$ was monitored and shown in figure 10 . The $\mathrm{OD}_{450}$ increased with the concentration of the coating conjugate from 2.5 to $10 \mu \mathrm{g} \mathrm{mL} \mathrm{m}^{-1}$, and reduced with the concentration increased to $20 \mu \mathrm{g} \mathrm{mL} \mathrm{m}^{-1}$. Thereby, a concentration of $10 \mu \mathrm{g} \mathrm{mL}^{-1}$ was used for the coating dextran-BSA conjugate.

Optimization of the incubation time: At temperature of $37^{\circ}$, the effect of incubation time of coating antigen reacted with polyclonal antibody and with sheep anti-mouse-IgG-HRP antibody was demonstrated in figure 11 . The result shown that the $\mathrm{OD}_{450}$ was most sensitive when the blocked coating antigen incubated with either polyclonal antibody or enzyme-labeled antibody for $45 \mathrm{~min}$.

Optimization of the blocking solvent: Two kinds of blocking solvent were investigated, including $5 \%$ BSA protein and $5 \%$ casein protein, the effect of washed and unwashed the plate was also studied. The results were demonstrated in Figure 12, and 5\% BSA protein as blocking solvent and the plate was washed three times after blocking was confirmed.

Optimization of the antibody dilutability: The effect of antibody dilutability was studied and the result shown in figure 13. The best dilutability was set as 1/32000 according to the result.

\section{Establishment of Elisa method}

Linear range quantitative analysis and lower limit of dextran T40: Using the optimized indirect non-competitive Elisa condition, a general and broad class-specific indirect competitive Elisa detection method was developed according to Elisa theory. With T40-BSA as coating antigen, concentration of $10 \mu \mathrm{g} / \mathrm{mL}$, polyclonal antibody dilutability of $1 / 32000$, a working curve for dextran T40 detection was acquired, the regression equation was $\mathrm{Y}=1.0969-0.5203 * \log (\mathrm{X})\left(\mathrm{r}^{2}=9796\right)$, with the linear range was $0.5-128 \mathrm{ng} / \mathrm{mL}$ and $\mathrm{IC}_{50}$ value as $32 \mathrm{ng} / \mathrm{mL}$, the limit of detection of $0.5 \mathrm{ng} / \mathrm{mL}$. The Elisa detection to other dextran (T10, T100, T2000) was also studied. (figure 14)
Specificity analysis: Using different dextran-BSA antigens (T10,T100 and T2000) as coating antigen, pure rabbit blood as the positive control, the corresponding dextran Elisa detection working curve were obtained as figure 14 . The $50 \%$ inhibitory concentration $\mathrm{IC}_{50}$ were gained respectively, and the cross-reactivities were calculated and listed in table 1.

The working curve shown different molecular dextran can be detected by T40 dex-BSA polyclonal antibody using Elisa detection. The higher dextran molecular gain the more sensitive the detection reached. From the cross-reactivity data, different molecular dextran hindered the Elisa detection of dextran T40. Dextran with similar molecular with dextran T40 had prominent effect. In consideration of dextran had similar structure, the developed method can be used for total dextran determination in practical samples.

\section{Application to practical samples}

Sucros samples were purchased from Guangzhou market, and the dextran determination was carried out according to the developped Elisa detection method. Different sample can be detected with the convenient method rapidly and credibly. One of the sample was shown as figure 15, according to dextran T40 detection regression equation, the dextran concentration was $3.5 \mu \mathrm{g} / \mathrm{mL}$ when the practical saccharose samples concentration was $25 \mu \mathrm{g} / \mathrm{mL}$.

\section{Conclusions}

In summary, dextran-BSA antigens were prepared by reductive amination method and the preparation condition were optimized as dextran oxidized by $\mathrm{NaIO}_{4}$ at the weight ratio of $1 / 120$ for 24 h. The immunocompetence of resulting antigens was evaluated by Elisa detection with commerce antibody, and rabbits were immunized by the screened dex-BSA T40 antigen to gain polyclonal antibody. An indirect Elisa detection method was developed for dextran determination, and the method with good linear range and sensitivity which was applied to dextran determination in practical saccharose samples.

\section{Acknowledgements}

We acknowledge financial support from the integration of the Tertiary Teaching, Research and Production foundation and 211 development foundation of the Science Research Fund Institutions of Guangdong province (China) for this project. Technical supports from Mr. Dong Zhining and Zeng Lianqiang were also greatly acknowledged.

\section{References}

[1]. Newman B.A, Kabat, E (1985) An immunochemical study of the combining site specifies of C57BL/6J monoclonal antibodies to $\alpha$ (1-6)-linked dextran B512. Journal of Immunology 135(2):1220-1231.

[2]. Efraín Rodríguez Jiménez (2005) The dextranase along sugar-making indus- 
try. Biotecnología Aplicada 22: 20-27.

[3]. E.A. Abdel-Rahman (2008) Influence of dextran concentrations and molecular fractions on the rate of sucrose crystallization in pure sucrose solutions. Journal of Food Engineering 84: 501-508

[4]. Elvira Khalikova, Petri Susi, Timo Korpela (2005) Microbial Dextran-Hydrolyzing Enzymes: Fundamentals and Applications Microbiology and Molecular Biology Reviews 69(2): 306-325.

[5]. Chung C.C (2000) Handbook of Sugar Refining [M]. New York: John Wiley \& Sons, Inc 516-517.

[6]. Day D.F, Cuddihy J.A, Rauh J.S (2002) Versatility of the antibody dextran test method. [M]. 32nd Annual Joint ASSCT Meeting, Amelia Island Plantation, FL 26-28.

[7]. Clarke M.A, Godshall M.A (1988) Determination of dextrans in raw cane sugar by Roberts copper method: Collaborative study. Journal-Association of official analytical 71(2): 276-279

[8]. Sarkar D, Day DF (1986) Dextran analysis: A modified method. J. Am. Soc. Sugar Cane Technology 6:102-107.

[9]. Singleton, V (2002) A new polarimetric method for the analysis of dextran and sucrose. Journal American Society of Sugarcane Technologists 22: 112 119.

[10]. Bo Mattiasson, Kosin Teeparuksapun, Martin Hedström (2010) Immunochemical binding assays for detection and quantification of trace impurities in biotechnological production. Trends in Biotechnology 28(1): 20-27.

[11]. Shavej A (2006) Dextran, 5-aminosalicylic acid (5-ASA) conjugates:synthesis, characterization and enzymic hydrolysis. Carbohydrate Research 34: 26942701.

[12]. Walaisiri M, Lee E. Kirsch (2006) The protein-binding and drug release properties of macromolecular conjugates containing daptomycin and dextran. International Journal of Pharmaceutics 315: 30-43.

[13]. Michael Böcher (1997) Synthesis of mono- and bifunctional peptide-dextran conjugates for the immobilization of peptide antigens on ELISA plates: properties and application. Journal of Immunological Methods 208: 191202

[14]. Ashraf Brik (2010) New Strategies for Glycopeptide, Neoglycopeptide and Glycoprotein Synthesis. Chemistry and Biology 6: 55-89.

[15]. Yoann M. C, René R (2010) Design and Creativity in Synthesis of Multivalent Neoglycoconjugates. Carbohydrate Chemistry and Biochemistry 63: $165-393$.
[16]. Laemmli U.K (1970) Cleavage of strucyural protein during the assembly of the head of bacteriophage T4. Nature 227: 680-685.

[17]. Zacharius R M (1969) Analytical. Biochemistry 30:148-154

[18]. Nielsen P M, Petersen D, Dambmann C (2001) Improved method for determining food protein degree of hydrolysis Food Science 66(5): 642-646

[19]. Tijssen P (1985) In: BurdenR.H, Van Knippenberg P.H, Laboratory Techniques in Biochemistry and Molecular Biology, vol. 15, Practice and Theory of Enzyme Immunoassays. Elsevier, Amsterdam. 